\title{
Special issue on recent progress in nonlinear theory and its applications
}

The International Symposium on Nonlinear Theory and Its Applications (NOLTA 2018) was held at Tarragona, Spain during September 2-6, 2018 with great success. This special issue is associated with NOLTA 2018. During the Symposium, researchers from all over the world including many students participated in the symposium and their excellent research activities from the various topics in nonlinear science and engineering ware presented. This special issue has been organized as a section associated with NOLTA 2018. However, the paper submissions were open for not only the authors of NOLTA 2018 but also for others who work with the related topics. We received 20 papers for this special issue and 15 papers were accepted after rigorous review processes. We also announce that 2 invited papers from Prof. Kunihiko Fukushima and Prof. Shun-ichi Amari were included.

We would like to express our sincere appreciation to the authors who submitted their manuscripts. Also, we thank to all the editorial committee members and anonymous reviewers for their great effort to this special issue. Finally, we would like to express our special thanks to Prof. Shigeki Shiokawa, who is the secretary of this special issue, for his hard work for this issue.

\section{Luis Martinez-Salamero and Seiichiro Moro Guest Editors}

\section{Editorial Committee of the Special Issue}

\author{
Guest Editors: \\ Luis Martinez-Salamero (Universitat Rovira i Virgili) \\ Seiichiro Moro (University of Fukui)

\section{Guest Associate Editors:} \\ Luis Benadero (Universidad Politecnica de Catalunya) \\ Federico Bizzarri (Politecnico di Milano) \\ Daniele Fournier (INSA-Toulouse) \\ Wataru Kurebayashi (Shiga University) \\ Takafumi Matsuura (Nippon Institute of Technology) \\ Gerard Olivar (Universidad Nacional de Colombia) \\ Toshimitsu Ushio (Osaka University) \\ Yoko Uwate (Tokushima University) \\ Naoki Wakamiya (Osaka University) \\ Secretary: \\ Shigeki Shiokawa (Kanagawa Intstitute of Technology) \\ Advisory:
Hiroo Sekiya (Chiba University)
}

